\title{
Principles and Practice of Work Style Modeling: Sketching Design Tools
}

\author{
Pedro Campos and Nuno Jardim Nunes \\ LabUSE - Laboratory for Usage-Centered Software Engineering \\ Dep. of Mathematics and Engineering, University of Madeira \\ Campus Universitário da Penteada, 9000-390 Funchal - Portugal
}

\begin{abstract}
In our research, we have been combining Work Style modeling with the well-established principles of Usage-Centered Design having the objective of designing and evaluating better design tools. Our approach distinguishes itself from the fact that it combines work style quantitative data (easily obtained through logging tools) with qualitative data that predicts a given tool's level of acceptance. We describe a set of principles that were proven successful during this design process, illustrate sketches of the tools, and highlight the relevant design aspects that worked and those that didn't work.
\end{abstract}

\section{$9 \quad 1$ Introduction}

Trying to promote a better understanding of the relationship between work-domain based experimental studies and iterative design of prototypes can only be achieved by designing new methods and models that are more useful and usable. We have designed a framework and a model (that we call the work style model) as an aid to the complex task of designing tools that support the equally complicated task of interaction design itself.

The work of interaction designers is very multifaceted: designers need practical guidance (not canned solutions), as well as concrete principles. Good models and good tools should highlight opportunities for innovation, leave the details open (concentrating on essentials), invite creative projection and inform - and guide towards good design. Although some research has been dedicated to examining why modeling tools are not used [1] or creating frameworks that can measure the tools usability in a cost-effective way [2], our approach distinguishes itself from the fact it combines work style quantitative (easily obtained through logging tools) with qualitative data that predicts a given tool's level of acceptance.

The remaining of this paper is organized in the following way: Section 2 briefly describes related research on work domain analysis, and models and tools for the interaction design tasks. Section 3 defines a new framework for studying the work of interaction designers as well as our work style model. It also describes the foundations of the framework as well as why it was designed that way. Section 4 presents the models and sketches of the design tools we have been building and evaluating (namely CanonSketch and TaskSketch). During this process of design and evaluation, we have collected and summarized a set of heuristic principles that

Please use the following format when citing this chapter:

Campos, P., Nunes, N. J., 2006, in IFIP International Federation for Information Processing, Volume 221, Human Work Interaction Design: Designing for Human Work, ed. T. Clemmensen, Campos, P., Orngreen, R, Petjersen, Al., and Wong, W., (Boston: Springer), pp. 203-219. 
proven useful. Those principles are presented in Section 5, which also describes which design aspects worked and which design issues didn't work. Finally, Section 6 briefly outlines our conclusions.

\section{Related Research}

Human Work Interaction Design is a recent research area which was born under the auspices of IFIP's Working Group 13.6. In a recent workshop [3], new themes and directions of research on human work analysis and design to support it have been outlined. The main target of the work group is the analysis of and the design for the variety of complex work and life contexts found in different businesses. Software design (which includes interaction design) is often a team activity and most projects involve stakeholders with different backgrounds that must cooperate in many different and interrelated activities, so it fits very well into the Working Group's directions.

Complex work activities increase the difficulty of predicting the level of acceptance of novel technology and how it will be used in practice. An important and open research question is how to translate usability evaluation results into concrete design decisions $[4,5]$.

The Technology Acceptance Model (TAM) developed by Davis and colleagues [6] is a widely used theoretical model in the Management Information Systems (MIS) field. Basically, it attempts to predict and explain computer-usage behavior, offering both researchers and practitioners a direct, pragmatic instrument to measure a technology's degree of acceptance. Morris and Dillon [4] pointed out that TAM offers HCI professionals a "theoretically grounded approach to the study of software acceptability that can be directly coupled to usability evaluations".

Wu and Graham [7] describe a novel model for recording the working style of people using an interactive system. Workstyle modeling complements task modeling by providing information on how people communicate and coordinate their activities, and by showing what style of artifact is produced.

The workstyle model was developed in the context of the Software Design Board project, a project aiming to provide better tools for software design. The model was validated through evaluation of existing design tools, and motivated the design of a new software design tool. It is comprised of eight axes: four of them describe collaboration style (Location, Synchronicity, Group Size and Coordination); the remaining four describe the nature of the artifact being produced (Syntactic Correctness, Semantic Correctness, Archivability and Modifiability).

The workstyle model for software design has the advantage of being simple to apply and clearly showing where a tool can fail to match the intended work context. However, it is not sufficient for capturing UI specific activities. Transitions (or shifts) in the workstyles of interaction designers are more frequent and more intense than in any other software design activity. This was the starting point of our investigation towards building a framework for interaction design activities, which we will describe in the following Section. 


\section{Styles for Work Styles: Definition of the Framework}

Interactive Systems Design methodologies, such as [8], often describe users in context by using the concept of actors. Usage-Centered Design (UCD) [9], for example, separates the actors of a system from the roles they play during the system's usage. Indeed, users adopt several roles during the usage of a system, just like film actors do, but they also switch roles throughout that usage. Although interaction design methods are well conceived to realize systems supporting the roles of usage, few methods provide support for flowing from different contexts/needs of usage.
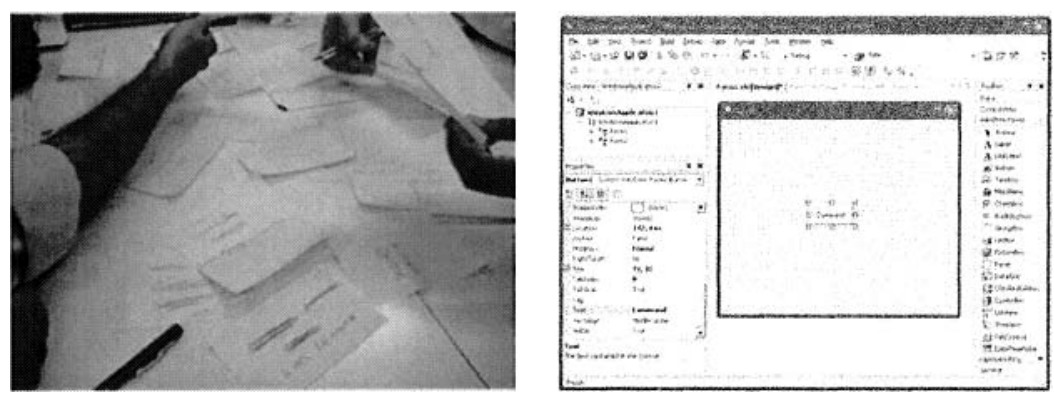

Fig. 51. A Work Style Transition: working in groups using low-tech materials for task modeling and clustering (left). After task modeling, each team member is assigned a set of tasks and builds concrete prototypes supporting those tasks using a visual interface builder (right).

Interaction Designers are the users of Design tools themselves, and in this context, we have developed and applied a model for describing the contexts in which they work, by modeling their Work Styles. A Work Style is an informally-defined set of values in $n$-dimensions. These dimensions describe the most important aspects of the way users work in order to achieve their tasks. A work style transition (or change) is a change in one or more values of a work style. A region (or plane) in a work style model is a set of work styles. Systems supporting work style regions are systems that can adapt to and support transitions in the users' styles of work. Figure 1 shows an example of a work style transition in the life of an interaction designer: on the left, a team of developers works together using post-it notes for task clustering in a spatially useful style. After this, the team splits and each designer is assigned a set of tasks and builds a concrete mock-up of the interface using an interface builder. Each designer transitioned from a low-detail, collaborative, low-tech work style to a high-detail, high-tech, individual work style.

\subsection{The Work Style Model}

The eight continuous axes in our Work Style model for UCD are shown in Figure 2. These axes are grouped under three main categories: 
- Notation style-related dimensions (Perspective, Formality and Detail),

- Tool usage style-related dimensions (Traceability, Functionality and Stability) and

- Collaboration style-related dimensions (Asynchrony and Distribution).

Each of these dimensions is described in [10], and for each dimension there is a set of questions that can act as guidelines aiding the process of work style classification. In this section, we briefly describe each of these dimensions and provide a set of questions that can act as guidelines to apply the model to tools, notations or, in general, styles of work adopted by interaction designers.

Perspective. This axis plots the perspective, or view, of the artifact being developed. Questions: is the notation capable of expressing business goals? Or nonfunctional requirements such as customer experience requirements? Does it help define the purpose of the system? Does it describe interaction aspects of the system? How close is it to the final product?

Formality. This axis classifies the work style of a designer creating artifacts in a formal vs. informal way. In the early stage of the process, designers use rough, ambiguous sketches to freely express ideas quickly [11]. This work style also fosters comparison of design alternatives and creativity, since the uncertainty of sketches encourages the exploration of design ideas. As design progresses, a more formal style of work is incrementally adopted, as designers need to focus on the precise meaning of their models. An example of this shift is moving from a whiteboard to a CASE tool.

Questions: how easy is it to define rough ideas? Does the meaning matter? Does the notation force you to use a rigid syntax/semantics?

Detail. We added this axis to plot the level of detail (or abstraction) the designer is working at. High-level, abstract models facilitate problem solving in organization, navigation and overall structure of the UI, leaving aside the details. On the other hand, realistic (or figurative) prototypes address high-detail design issues [12]. Disciplined designers tend to assume a work style that goes from higher-level abstract representations towards more realistic and detailed representations as the process evolves [12].

Questions: can you abstract irrelevant details using the notation? Can you think about navigation and structure of the overall interaction using the notation? Can you incrementally add enough detail?

Stability. This dimension describes how difficult/frequent it is to modify any aspect of the artifact(s) being developed. A content inventory of the UI modeled in a UML tool is highly modifiable because it is easy to change names, positioning, size and other aspects of the elements. This is opposed to drawing a model of the UI with pen and paper, since changes are harder to accomplish. Brainstorming, for instance, is a very unstable work style because changes are very frequent. High values in this axis indicate less frequent or less significant changes.

Questions: How easy is it to modify previously created artifacts using the tool? How frequently do you make those changes? Are there particular changes difficult to accomplish with the tool?

Traceability. This is a new dimension we introduce. It describes if the elements of the artifact being developed are consistent and interconnected (thus being highly traceable) or if they are completely unrelated and independent. As an example, 
developers might adopt a work style in which they choose to keep links from task cases steps and the concrete UI widgets that implement those task steps. In this case, it is possible to trace a task step to the concrete widget and to trace a widget to the task step it implements. This dimension is closely related to stability and the number of artifacts produced during a project. As they increase, traceability becomes more important.

Questions: Are you using the tool to maintain interconnections between model elements? How important is it to navigate through your model? Does the tool maintain several different views in a synchronized way (e.g. design view and code view)?

Functionality. This is also a new dimension we introduced. It represents how much functionality is being addressed (by using the tool to build a prototype). There is a barrier between software engineers and usability professionals regarding this matter: software engineers are engaged into building reliable, functional systems, leaving user-friendliness to the usability specialists. Usability and interaction designers, on the other hand, first design and test the interface with end-users, leaving implementation to software engineers, regarded as functionality builders. Those two processes should not be separated [13] and considering this dimension will help overcome that barrier. This dimension is also important because designers combine visual design (presentation issues) with interaction design (behavior issues).

Questions: How much functionality, behavior and dynamics can you add to your prototypes using the tool? How easy is it to test the interaction by using the tool?

Asynchrony. This axis refers to the collaboration style that designers assume: they can make changes to the work being developed at the same time (a synchronous work style) or they can work at different times (engaging in an asynchronous work style) [7]. The higher the value in this axis, the more asynchronous is the work style.

Questions: do the team members change artifacts at the same time? Or do they make changes at different times? How frequently?

Distribution. This dimension describes whether work is being conducted at the same physical location or at geographically distant locations.

Questions: how far are the team members collaborating? Are they in the same building? Or are they in a different continent, or scattered through a country?

These dimensions can be effectively used to assess a given work style adopted by an interaction designer or a team. A single work style is plotted as a line (a point in the eight-dimensional space) whereas regions (or planes) represent sets of work styles.

Figure 2 also shows how this model was used to drive the development of a new user-centered tool for designing UI's (User Interfaces). CanonSketch $[14,10]$ is an UML-based tool that supports multiple levels of detail by providing the designer with three views: UML view of the UI and domain models, Canonical Abstract Prototype [12] and HTML concrete prototype (as the right side of Figure 2 exemplifies). The first two views are synchronized and the UML semantic model is used to support traceability. There is also a collaborative version of this tool in which designers can work at the same time on the model and at different places. However, support for distribution is still limited (for instance, there are no awareness mechanisms). Therefore, CanonSketch supports a region in our model, as illustrated in Figure 2. 
In this way, the tool seamlessly supports designers while switching from highlevel abstract views of the UI and low-level concrete realizations [14]. CanonSketch has been tested under a laboratorial setting and has lead to promising results. By contrast, a visual Interface Builder only supports a line in the work style model (the dashed line in Figure 2).

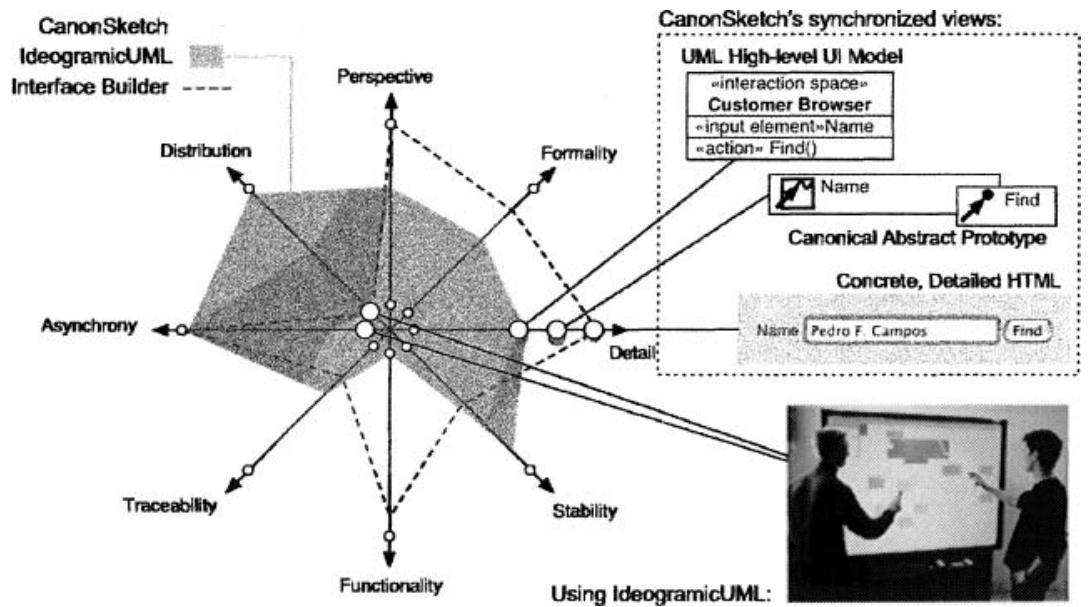

Fig. 52. Illustration of three different tools plotted under the Work Style Model.

IdeogramicUML, a gesture-based UML modeling tool, was also plotted in our model. It covers a considerable region, which suggests its adequacy to several styles of work during development tasks. IdeogramicUML only supports the syntax of the UML. However, it uses a sketch recognition language and can be effectively used in electronic whiteboards. There is also a distributed version with awareness mechanisms built in. Thus, one of the better supported transitions in work style provided by this tool is switching from synchronous, co-located development to asynchronous, distributed development.

\subsection{2 A framework for studying Work Style support}

In recent research results, we discovered that professional practitioners of interaction design engage into different work styles throughout their quotidian endeavors. We performed a survey which was distributed to professional interaction designers associations and mailing list, and collected 245 usable responses. This study, which is described in [15], had two main goals:

- Assess the practical aspects of the work style model: in particular if asking questions about work styles would be feasible and would lead to interesting findings; 
- Find interesting patterns of tools' use and/or work style transitions among industrial designers.

Among other issues, we were interested in finding out which work style transitions did the practitioners considered more frequent and more difficult, in their everyday work practices. By frequent, we meant "how many times [the respondent] engages and transitions in those work styles", and by difficult we meant "how difficult [the respondent] finds to perform that transition". We confronted respondents with several concrete scenarios of work style transition and asked them to rate frequency and cost by selecting a value from a 7-point Likert scale, labeled with 1-low, 4-moderate and 7-high. Figure 3 shows the average rate for each transition.

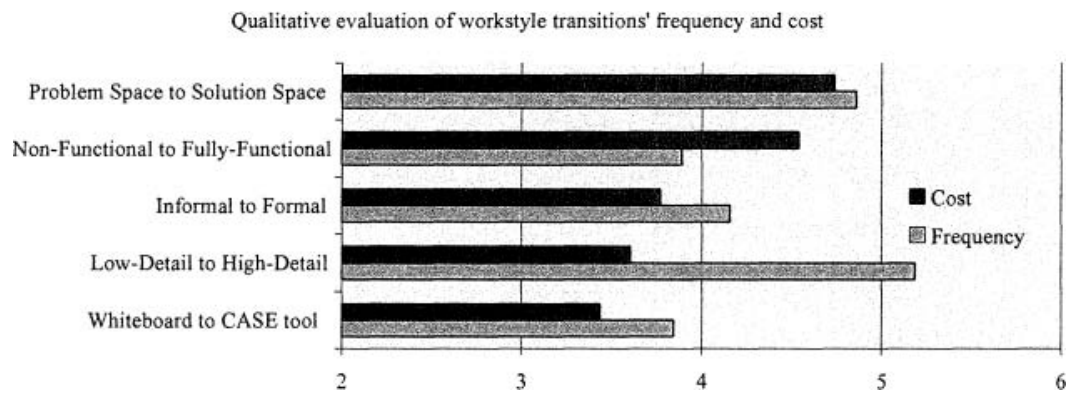

Fig. 53. Some transitions in work styles and their frequencies and cost.

Results showed that the most frequent transition was "moving from high-level descriptions of the user interface (sitemaps, navigation maps, etc.) to detailed screens (with concrete widgets, buttons, etc.)". This is a detail work style transition ("lowdetail to high-detail". The second most frequent transition was also rated the most difficult one: "Moving from business rules, use cases and problem space concepts into final solution design, and back". This is a perspective work style transition ("problem space to solution space").

Based on the Technology Acceptance Model [6], current research literature [7], the Workstyle Model for UCD [10] and our survey's results [15], we designed an experimental framework aimed at studying the interaction designer's tools and work styles. Figure 4 summarizes the constructs in our framework, as well as the hypotheses we tested.

Perception-related variables operationalize the constructs of this framework. Four perception-based variables are measured, just like in the TAM:

- Perceived Usefulness (PU) is defined as the degree to which the user believes that using the tool will enhance his or her performance in designing interactive systems;

- Perceived Ease of Use (PEOU) is defined as the degree to which the user believes that using the design tool will be free from effort; 
- Attitude toward using (A) measures the feelings of favorableness toward using the tool;

- Behavioral intention to use (BI) measures the strength of a designer towards using the tool in the near future.

Work Style-related variables measure some aspects that come from our Work style model and from the transitions considered most difficult and frequent by professional interaction designers (according to our survey):

- Perspective Transitions Frequency (P) is defined as the rate of transitions from different perspective views, i.e. the frequency of transitioning from problem space concepts (use cases, task flows) to solution space (architecture, abstract prototype) and back;

- Detail Transitions Frequency (D) is defined as the rate of "drill-down" or "rollup" between model elements, i.e. switching from high-detail views of an element to low-detail or the opposite;

- Modifiability Rate (M) is the rate of change made to any element of the artifact(s) being designed. This might include changing names, color, size, values or any other property of elements.

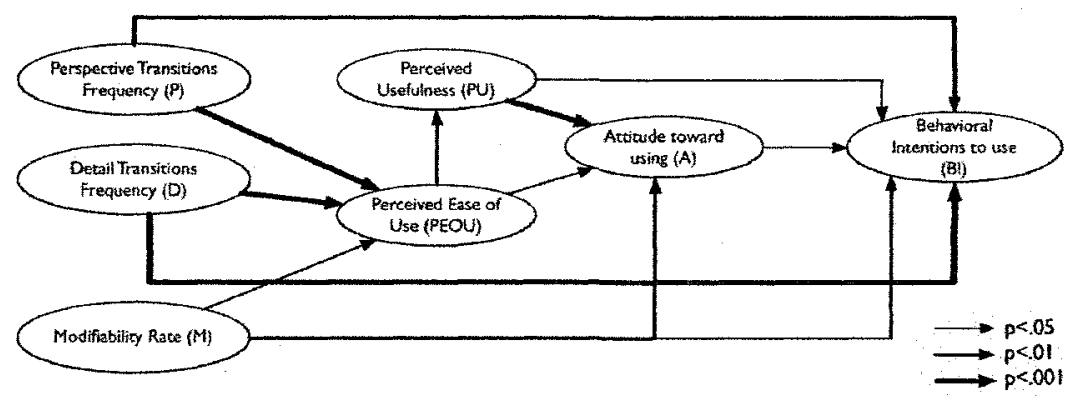

Fig. 54. Framework for combining Work Style analysis with Technology Acceptance analysis.

The framework was tested using logging tools for the quantitative measurements ( $\mathrm{P}, \mathrm{D}$ and $\mathrm{M}$ ) and a survey that measured qualitative responses to $\mathrm{PU}, \mathrm{PEOU}, \mathrm{A}$ and BI. Figure 4 shows the revised theoretical model after regression analysis. The stroke of the arrows' thickness depicts the statistical strength of the relationships between factors. We observed how work style transitions have an influence on the tools' perception of usability and usefulness as well as behavioral intentions to use it.

If perspective and detail transitions are viewed by professional interaction designers as the most difficult (perspective) and frequent (detail) kind of transitions, and if our results show that these transitions' frequency has negative impacts on the tools' perceptions and intentions of use, then tool designers should find innovative ways to ease those transitions. The same happens with modifiability: the results suggest that the more modifications, the lower the positive feelings regarding the tool. Since we have showed that almost $80 \%$ of the time is spent modifying artifacts, effort should be targeted at easing this activity. 
This framework of influences, together with our survey's instrument and Work Style model constitutes the core of our framework. We used it to design and evaluate a new set of design tools (CanonSketch and TaskSketch), aimed at improving the design experience and achieving more usable products.

\section{Sketching and Designing Design Tools}

We have been combining Work Style modeling with the well-established principles of Usage-Centered Design (U-CD) with the objective of designing and evaluating better design tools. In this section, we will present the interpretations and actual implementation of some of our most interesting designs.

\subsection{Usage-Centered Models and Sketches}

In this section, we describe some of the most interesting models, based on UsageCentered design principles we applied during the process of designing new tools.

Usage-Centered Design is a methodology that tries to get the right design right from the start [9]. The twin foundations upon which U-CD is built are process and principles. U-CD defines an orderly and efficient process to transition from problem space to solution space. The process is guided by principles of what is considered good design, design that is most likely to allow the users accomplish their goals. Neither the U-CD process or principles guarantee one will design a good solution, but following both process and principles will improve the odds.

The essence of U-CD lies at three core models: the user role model, the task model and the interface content model. The difference between this and other design methodologies is relying on abstract, technology-independent models which prevent the designer of getting lost in the details of the design or in technology commitments.

We combined U-CD process and principles with our work style analysis framework for designing new tools, i.e. we apply U-CD to the design of U-CD tools. concerning users, we are interested solely in the roles they play in relation to a system, and we capture the prominent and noteworthy aspects of these associations in the shape of an abstract user role model. Figure 5 shows the user role model coupled with the most significant tasks each role is related to.

We define a "General-Modeling Role" which is sometimes specialized into a "User-Roles Modeling Role", a "Task-Cases Modeling Role", "Content Modeling Role", and a "UI-Realizing Modeling Role". Each of these roles is assumed by the user (the interaction designer using the tool) and each of them represents a different position in the work style space. In our tools' design and implementation we have tried to give support to a fluid transition between these any of these roles. 


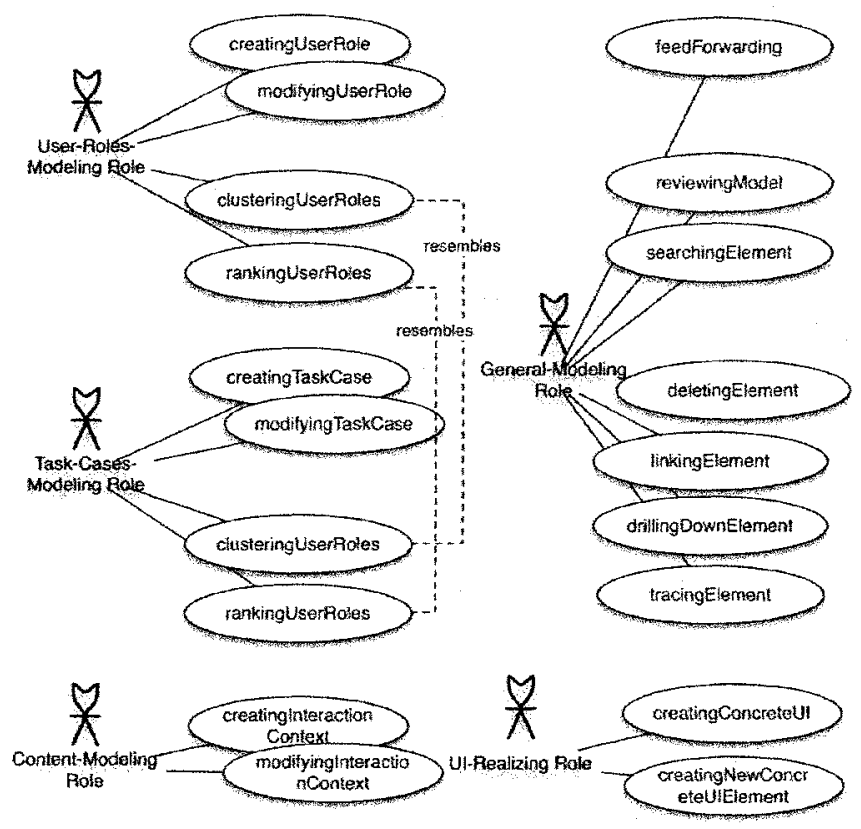

Fig. 55. User Role Model for a Usage-Centered Design Tool (full model omitted for brevity).

Another noteworthy aspect is related to the resemblance between some of the tasks. In empirical studies we discovered the designer is engaged into two main activities: creating (roughly 25\%) and modifying (roughly $75 \%$ ). Modifying a design also means reviewing it. This makes "Creating...", "Modifying..." and "Reviewing..." the central tasks in our model (although this is not explicit, we kept it in mind during the concrete, final design).

Figure 6 exemplifies how one proceeds to the task model. For a task model, we turn to task cases: these are use cases defined by abstract, generalized, technologyfree descriptions $[9,12]$. These can also give rise to interesting design ideas: as an example, consider the linking element task case. We declare that it is a system responsibility to adequately "show the designer available link types". As the user "chooses link type" (e.g. an aggregation, composition, etc.), the system should "provide feedback on valid sources". After choosing a valid source, the system should then "provide feedback on valid targets".

While there are many possible realizations of designs that support this task description, the truth is that it can be effectively realized in different platforms or even different modalities. Regarding different platforms, we only implemented it in the Mac OS X environment, but we are already working on a Windows design and implementation. Regarding different modalities, we are implementing it by using a pen and tablet system (using sketch recognition libraries) and by using voice input and output (simply using a speech recognition library and a microphone plus speakers). 


\begin{tabular}{|c|c|c|c|}
\hline $\begin{array}{l}\text { linkingEiement } \\
\text { User Intestions }\end{array}$ & System Responsfilitities & drillingDownElement & \\
\hline & showe arosidoble link types & User Intentions & System Responsibilitives \\
\hline \multirow[t]{2}{*}{ choose kink tope } & & choose madel siement & \\
\hline & Provide feedbock an wetid sources & Fequest a drill dewa & \\
\hline \multirow[t]{2}{*}{ choose source model tetemient } & & & provide detesiled info \\
\hline & provide feedtback on yolid targets & optismaliy request to edif & \\
\hline \multirow[t]{2}{*}{ chrose targot thodel elemines.t } & & & showe editing tcols \\
\hline & pitare link & godit elements & \\
\hline
\end{tabular}

\begin{tabular}{|c|c|c|c|}
\hline \multirow[b]{2}{*}{ tracingElement } & \multirow[b]{3}{*}{ System Responsibilities } & \multicolumn{2}{|l|}{ creatingConereteUl } \\
\hline & & User Enterticons & System Responsibalities \\
\hline User Intentions & & revip* task cases to support & \\
\hline choose model element & & revien tontent model & \\
\hline \multirow[t]{2}{*}{ request frace } & & choose obs, camponent to realizz & \\
\hline & show related models & & show possible realizothors \\
\hline \multirow[t]{2}{*}{ choose mondel to troce } & & Choose reotization & \\
\hline & show related efements in the model & & ploce it \\
\hline
\end{tabular}

Fig. 56. Some Essential Task Cases for Design Tools.

The main point here is that these abstract, essential descriptions help developers of design tools to focus on what is really important to users (in this case, knowing which sources/targets are valid dynamically). It also helps to be technology independent because we avoid early design decisions that could prevent us from exploring different designs. In the next section, we will describe concrete sketches of our design tools. The sketches were made using Apple's Interface Builder, and we tried to give support to the tasks in our U.CD model as well as to smoothly support the most significant and important work style transitions.

\subsection{CanonSketch and TaskSketch}

CanonSketch [10, 14] and TaskSketch [16] are two closely related innovative modeling tools that try to address major weaknesses in current model-based tools. They have proved to be effective in promoting innovative visual and interaction designs that better support user performance.

CanonSketch (available at http://dme.uma.pt/canonsketch) is the first tool to support abstract user interface prototyping using canonical abstract components. Canonical abstract prototypes serve as an intermediary between task and object models on the one hand and working user interface prototypes on the other.

The tool enables rapid modeling and prototyping through three synchronized views at different levels of abstraction: UML class model, canonical abstract prototype, and functioning HTML prototype. 


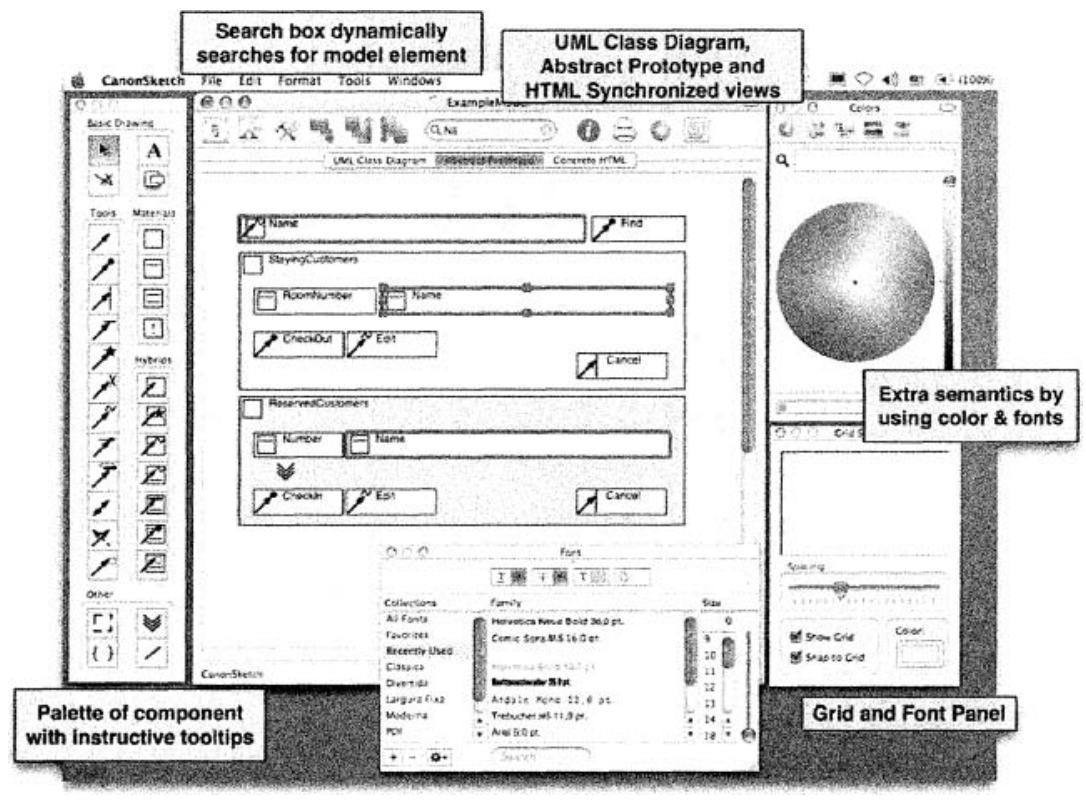

Fig. 57. CanonSketch illustrated.

Figure 7 illustrates a sketch of CanonSketch's main interface. The three synchronized views at different levels of abstraction try to support work style detail transitions. Since we showed that reviewing and searching a model significantly occupies the time of a designer, we provide a search box that highlights one or more model elements dynamically (i.e. as one types).

Extra semantics was also found to be important, because designers often extend the modeling language to convey extra meaning. This is done through using color and fonts (we are also implementing and testing a "star-rating" mechanism that helps designers classify the most urgent or important model elements).

TaskSketch (available online at http://dme.uma.pt/tasksketch) is an interactive requirements elicitation and modeling tool focused on linking and tracing use cases. It supports collaborative modeling by multiple stakeholders, including clients, marketing staff, and software engineers. It is unique in facilitating the development and exploration of the conceptual architecture based on use case narratives developed in essential form. It enables tracing the requirements of a system, in terms of user intentions and system responsibilities, to the conceptual architecture of that same system, making it easy to extract that architecture from task flows and to prioritize development of the most important classes. 


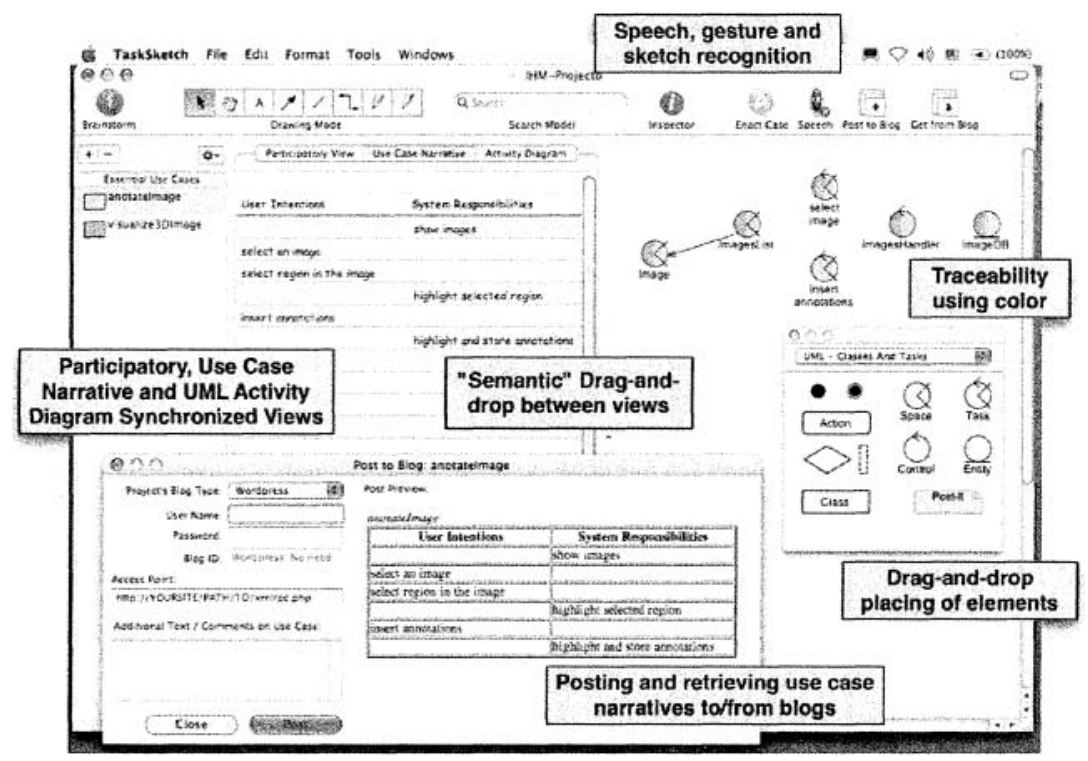

Fig. 58. TaskSketch illustrated.

Figure 8 illustrates some aspects of this tool. Traceability between related model elements is achieved by using color; the developer can look at the architectural view of the system and see which classes handle which use cases. This simple support to requirements traceability can be very powerful for, e.g., prioritizing development by deciding which classes are more urgent to implement. TaskSketch also supports what we call a "semantic drag-and-drop" between views. For example, if the designer drags a user intention from the use case narrative view and drops it into the architecture view, the tool creates the corresponding architectural element (in this case a task).

Following the same spirit of CanonSketch, this tool also provides the designer with three synchronized views for describing use cases and task steps. The Participatory view that uses post-it notes is good end-users and clients. The Task Case Narrative view is targeted at Usage-centered designers, and is nothing more than a digital version of index cards commonly used during this process. The UML Activity Diagram view is adequate to software engineers that practice sound, rigorous development methods such as the Rational Unified Process. Each user of TaskSketch can thus choose it's preferred work style and stick to it having the possibility of transitioning to another style whenever desirable (a software engineer working using the UML view can rapidly switch to the Participatory view to communicate models to a client or end-user).

Finally, we are also concentrating effort on exploring the possibilities offered by gesture recognition, mixing formal and informal notations and collaborative development using speech recognition and a shared display. This accounts as an effort to support collaboration styles and formality transitions. 


\section{Principles and Practice}

In designing CanonSketch and TaskSketch, many issues were raised both from informal observation of the tools' usage and from formal evaluation studies in laboratorial settings. We will try to summarize a few of the most relevant issues, starting with "what worked well", then describing "what didn't work well".

\subsection{What worked?}

"Semantic" drag and drop. This idea came up as a means to support the Traceability and Perspective dimensions. Designers can drag elements from the activity diagram view (that describes task flows) and drop them in the architecture view, where they are translated to their semantic equivalent. This feature lowered the cognitive load designers were faced when switching views or perspectives and was very appreciated.

Blogs as use case repositories. We wanted to better support collaboration work styles, so we used blogs as common use case repositories fostering offline collaboration (different time, different places). Users can post comments, make changes using web browsers and simply browse the use case narratives, and then designers can choose one or more blog posts and retrieve them into the semanticallysound TaskSketch models. Figure 8 illustrates this mechanism.

Brainstorm Collaborative Environment. Also inline with supporting collaboration work styles, we wanted to give users a tool to work at the same time but at different places. The brainstorm collaborative environment, where users concurrently post and cluster task cases, classes or UI ideas, proved very attractive to users, probably because of its' Messenger-like interface or the dynamics of graphics.

Dynamic Search Box. The dynamic search box that smoothly highlights model elements as the user types proved very useful not only as a search means but also as a way to "filter" desired elements (sharing common properties from the designer's perspective).

\subsection{What didn't work?}

Tool palette as opposed to Drag-and-Drop palette. CanonSketch used a Tool Palette "a la" MS Paint, which users tended to dislike (although appreciating the tooltips). Following users' suggestions we implemented a drag-and-drop palette ("a la" MS Visual Studio) in the TaskSketch tool. Apparently, users found this approach more usable.

Lack of automatic alignment. Clearly these design tools need to improve or adopt automatic alignment mechanisms of some sort. As we have shown, high modifiability rates negatively influence one's attitude toward adopting a design tool, so it is imperative to add this concern when deploying tools for designers. Simply drawing the model elements on the screen, or having a grid layout like MS Visio won't do, 
Use Cases manipulation view. Users didn't find comfortable using the "tablelike" interface for creating and manipulating the use cases. This is probably related to the process itself: use cases are the central constructs from which everything else is modeled, but they need to be effectively clustered and manipulated.

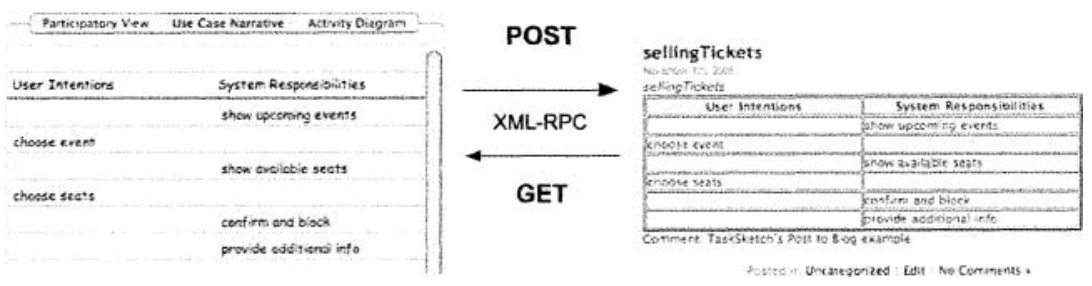

Fig. 59. The "posting to/getting from" blog mechanism illustrated.

\subsection{Principles based on Work Style Analysis}

Based on what we learned during the design and evaluation of both CanonSketch and TaskSketch during a 2-year period, we suggest - and in some cases we try to illustrate - a set of concrete design principles for tool developers.

Explorability. Design tools should make it easy to explore design alternatives. Basic undo-redo mechanisms are a poor man's approach to support this. Designers should not be penalized for trying out other models/solutions and not being able to go back or change ideas. What the Work Style model also suggests is that tools should invite creativity in early design stages and force focus and discipline later on.

Expressiveness. One of the major areas of weaknesses in current modeling tools is related to its' expressive power. A significant skill required to interaction designers is the ability to express and present their design ideas. Tools should provide helpful mechanisms to achieve this.

Guidance. Depending on the notation being used, the design tool should "justenough" constrain the actions of the designer. Designers need efficient guidance also on the form of concrete design principles, not canned solutions.

Desirability. Models created with the tools should look engaging and attractive. The user interface should be stylish. Designers are more prone to adopt a design tool based on the visual design of the models created with that tool. A desirable and engaging user interface

In general, we believe that a tool supporting regions of our work style model will clearly aid the design and analysis tasks faced by developers of interactive products. Providing support for multiple levels of detail and formality enables designers to start with low-fidelity sketches of products, then gradually add the precision and detail needed to test a prototype. The flexibility achieved by such a tool will most likely contribute to the ease of learning of both tool and method, accommodating to the user's work style and usage impulses. 


\section{Conclusions}

Work style modeling has proven a successful, practical method (when coupled to UCD) of designing a new set of design tools. Our framework has been used not only to design but also to evaluate design sketches and analyze the work practices of interaction design, both at academia and industry.

There are many advantages that arise from work style modeling. Supporting work style transitions is a good way to increase the odds of sketching solutions that provide a tighter fit between everyday work practices and the system being developed. Our work style framework also informs design: thinking about the transitions between values in work style dimensions can give rise to new design ideas and serve as a means to validate design decisions.

Describing and modeling the styles of work users were engaged with has helped us design more usable UCD tools, such as CanonSketch and TaskSketch. Usability studies have shown better results and we believe work style modeling can be very useful both as an informal discussion tool and as a Human-Work Centered Design approach.

\section{References}

1. Ivari, J. (1996). Why are CASE Tools Not Used? Communications of the ACM, 39:94-103.

2. Seffah, A. and Kline, R. (2002). Empirical Studies on Software Developers' Experiences. In WESS'02: Eighth IEEE Workshop on Empirical Studies of Software Maintenance. October 2nd, 2002. Montreal, Canada.

3. Clemmensen, T., Orngreen, R. and Pejtersen, A. M. (2005). Describing Users in Contexts Perspectives on Human-Work Interaction Design. Workshop Proceedings of Interact'05, Rome, Italy.

4. Morris, M. G. and Dillon, A. (1997). How User Perceptions Influence Software Use. IEEE Software, 14:(4), 58-55.

5. Wright, P., Dearden, A. and Fields, R. (2000). Function Allocation: A perspective from studies of work practice. International Journal of Human Computer Studies, 52:(2), 335356.

6. Davis, F. D. (1989). Perceived Usefulness, Perceived Ease of Use and User Acceptance of Information Technology. MIS Quarterly 13:(3).

7. Wu, J. and Graham, T. C. N. (2004). The Software Design Board: a Tool supporting Workstyle Transitions in Collaborative Software Design. In Proceedings of the EHCI / DSV-IS'2004, Hamburg, Germany.

8. Nunes, N. J., Cunha, J. F. (2001). WISDOM: Whitewater Interactive System Development with Object Models. In Mark van Harmelen (Editor), Object-oriented User Interface Design, Addison-Wesley, Object Technology Series, 2001.

9. Constantine, L. and Lockwood, L. (1999). Software for Use. A Practical Guide to the Models and Methods of Usage-Centered Design. Addison-Wesley, Reading, MA, pp. 194195.

10. Campos, P. and Nunes, N. J. (2005). Galactic Dimensions: A Unifying Workstyle Model for User-Centered Design. In Lecture Notes in Computer Science, Volume 3585, pp. 158169.

11. Landay, J. and Myers, B. (2001). Sketching Interfaces: Toward More Human Interface Design. IEEE Computer, pages 56-64. 
12. Constantine, L. (2003). Canonical Abstract Prototypes for Abstract Visual and Interaction Design. In Jorge, J., Nunes, N. and Falcão e Cunha, J. (eds.), Proceedings of DSV-IS'2003 - 10th International Workshop on Design, Specification and Verification of Interactive Systems, LNCS - Lecture Notes in Computer Science. Springer-Verlag.

13. Seffah, A. and Metzker, E. (2004). The Obstacles and Myths of Usability and Software Engineering. Communications of the ACM, 47(12): 71-76.

14. Campos, P. and Nunes, N. J. (2004). CanonSketch: a User-Centered Tool for Canonical Abstract Prototyping. In Proceedings of the EHCl/DSV-IS'2004, Hamburg, Germany, 2004.

15. Campos, P. and Nunes, N. J. (2006). Tools of the Trade: the Practitioner's Tools and Work Styles. Submitted for Review.

16. Constantine, L. and Campos, P. (2005). CanonSketch and TaskSketch: Innovative Modeling Tools for Us-age-Centered Software Design. In Conference on Object Oriented Programming Systems Languages and Applications (OOPSLA'05), Companion to the 20th annual ACM SIGPLAN conference on Object-oriented programming, systems, languages, and applications, San Diego, CA, 162-163. 